\title{
Traction control and diagnostics of electric and unmanned vehicles on roller stands
}

\author{
Alexander Fedotov ${ }^{1}$, Oleg Yankov $^{1 *}$, and Anton Chernyshkov ${ }^{1}$ \\ ${ }^{1}$ Irkutsk National Research Technical University, 83, Lermontov Street, 664074, Irkutsk, Russia
}

\begin{abstract}
The authors of the article analyze the possibilities of correct diagnostics of unmanned and electric vehicles on modern power and inertia roller stands. The purpose of this work is to improve the quality of monitoring the technical condition of wheeled vehicles by providing highly informative and stable test modes of operation in the process of their diagnostics. To achieve this target, we have developed test methods for wheeled vehicles, the implementation of which it is proposed to use a hybrid designed stand allows to measure force and power to the drive wheels of the vehicle, and temporal and kinematic parameters of the process of its functioning. The study proved that, to ensure a highly stable and test modes of operation of wheeled vehicles in the process of control of technical condition and diagnosis, you should use hybrid stands.
\end{abstract}

\section{Introduction}

Our country's highways are actively developing electric vehicles and unmanned vehicles are already appearing. Experience suggests that the number of such cars will only grow every year. Therefore, in the very near future, there will be urgent issues of maintaining and restoring their performance, providing high-quality diagnostics. There are known studies aimed at studying the diagnostics of battery system failures in electric and hybrid cars [1-7]. However, it is necessary to study the traction and speed properties of such vehicles. Unmanned wheeled vehicles are silent. They do not have a driver who could formulate questions about the technical condition of wheeled vehicles, evaluate its traction-dynamic and braking properties, describe the problems of its units, components and systems.

Electric vehicles and vehicles are characterized by both the classic typical layout of the power unit and transmission, and the layout with spaced electric motors individually on each driving wheel. In the first case the supply of torque to the driving wheels of the vehicle is carried out by a single electric motor through a reducer and hinges of equal angular speeds (fig. 1).

\footnotetext{
* Corresponding author: yos913005@mail.ru
} 


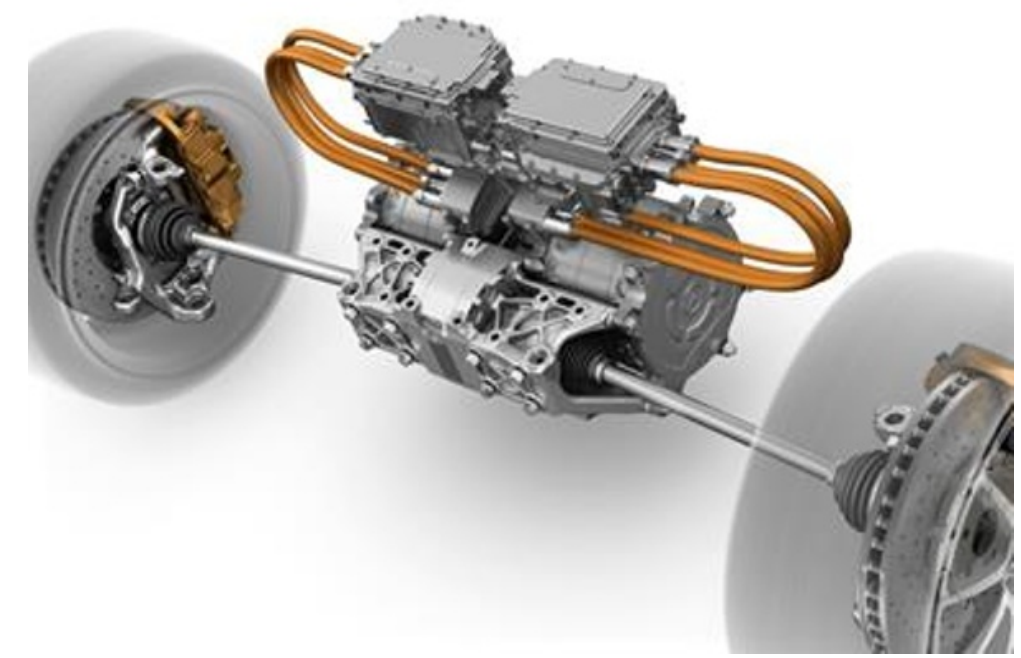

Fig. 1. Power units of electric vehicles with one electric motor per axle

In the second case, the electric motor is installed inside the driving wheel of the car (fig. 2). If monitoring the technical condition of electric vehicles with a single power unit is quite possible on existing stands, then for monitoring electric vehicles and unmanned wheeled vehicles with motor wheels, there is a problem of the lack of methods and means for monitoring the traction force, individually on each driving wheel. Modern bench diagnostic equipment does not provide this possibility.
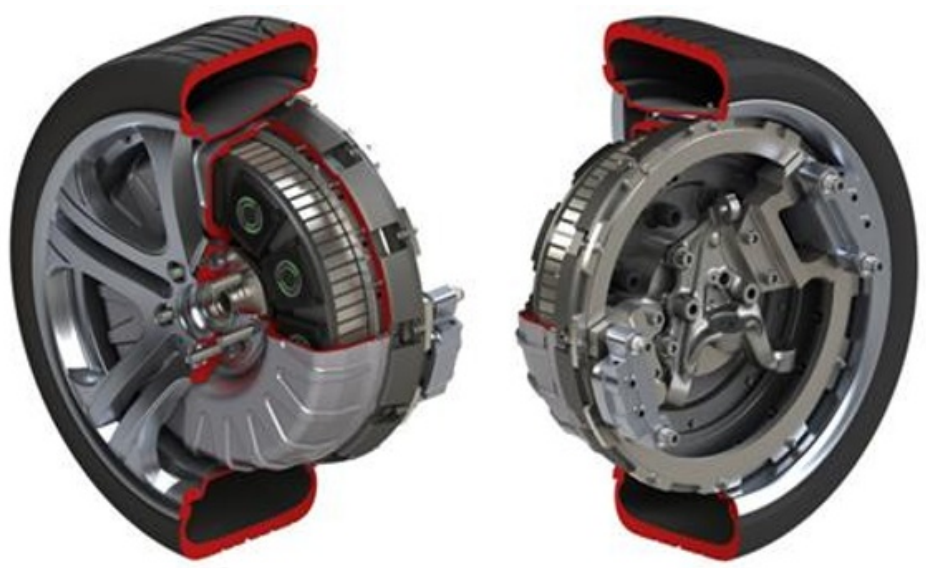

Fig. 2. Power units of electric vehicles with one electric motor per wheel

\section{Methods}

Not all stands with running drums and the methods they implement reflect the traction properties of cars correctly [8-10]. More or less correct and informative parameters from the diagnostic point of view are provided by power stands. They allow you to measure power parameters: traction, power on wheels, power and power spent on scrolling the transmission.

Modern power stands (fig. 3) consist of blocks with support rollers 2 and supporting rollers 5 . The support rollers 2 are connected to each other by couplings, as well as to the loading device 6, which is an electrodynamic brake. Sensors 1 track the speed of the car's 
wheels. The force with which the electrodynamic brake 6 tends to load the car's transmission is determined using the sensor 7. Sensor signals are processed and the power unit 9 and the amplifier and converter unit 8 control the stand.

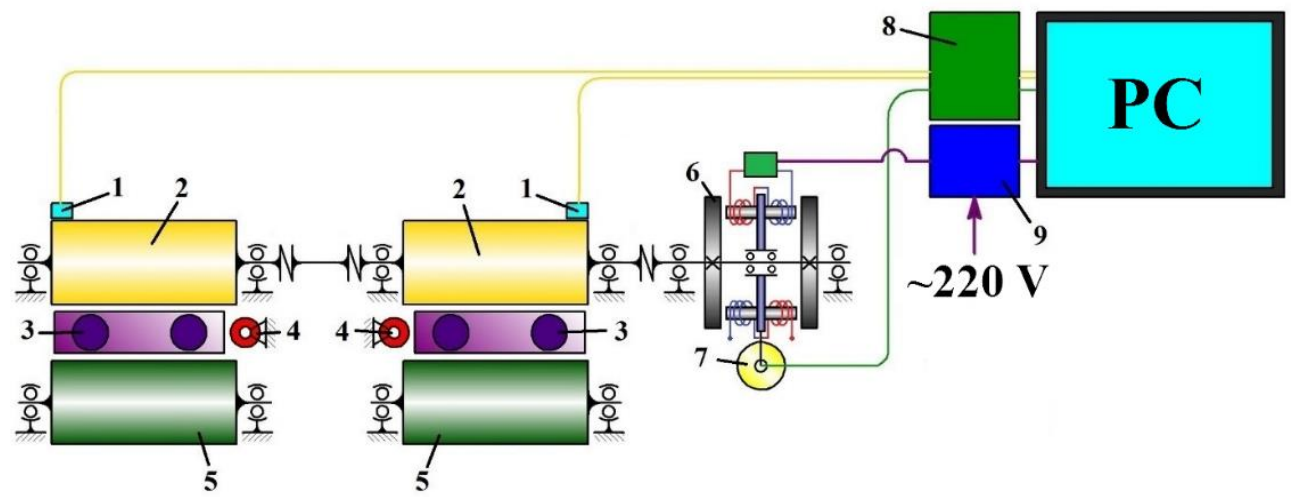

Fig. 3. Kinematic diagram of the power stand:

1-speed sensor; 2-support roller; 3-lifting device; 4-bump roller; 5-supporting roller;

6-electrodynamic brake; 7-force sensor; 8-unit of amplifiers and converters; 9-power unit.

Unfortunately, when monitoring cars on power stands, it is possible to obtain their characteristics only in a steady mode, for example, to simulate the movement of the car at a constant speed up the hill. Although in reality, the movement of the car is mostly accompanied by acceleration and run-out modes. Most faults can be detected in these modes. Therefore, for a complete and informative diagnosis of wheeled vehicles on stands with running drums, it is necessary to simulate the acceleration of the car. This cannot be done on power traction stands.

The direct opposite of power stands is inertial stands that implement dynamic methods of diagnostics of wheeled vehicles. Inertial stands (fig. 4) consist of blocks with supporting rollers 3 and supporting rollers 6 . Each support roller 3 is connected by a coupling to the reducer 1 , which, in turn, is connected to the flywheel 7 , which performs the functions of a loading device. The speed of the car's wheels is tracked by 2 sensors. Sensor signal processing and control of the stand is carried out by the amplifier and Converter unit 8 and the power unit 9.

Unlike power stands, they allow you to measure only time and kinematic parameters, such as acceleration time, acceleration path, run-out time, and run-out path. Analysis of the design of the traction quality stands shows that there are also inertial power stands that implement the power and inertial methods for setting test modes. The force parameters are measured using a force sensor mounted on a balancing eddy current brake.

All of the above stands are able to measure the total traction force on the driving wheels of the car. While modern electric vehicles and unmanned wheeled vehicles need to control the traction forces on each wheel individually. 


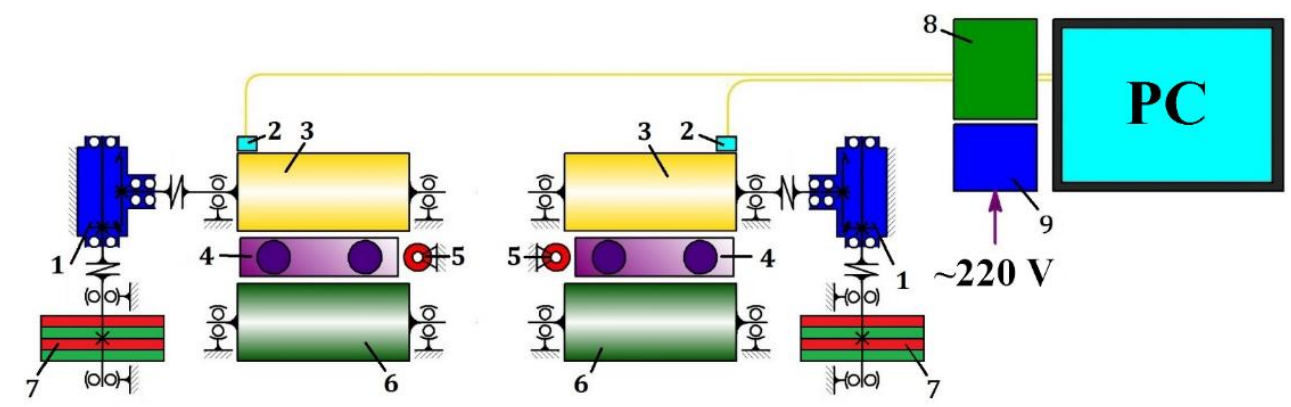

Fig. 4. Kinematic diagram of the inertial stand:

1-reducer; 2-speed sensor; 3-support roller; 4-lifting device; 5-rebound roller; 6-support roller;

7-flywheel; 8-unit of amplifiers and converters; 9-power unit.

Measured on the inertial stands for the kinematic and temporal parameters are extremely unstable. They are not informative and require a large number of measurements. But at the same time, the dynamic methods implemented by these stands simulate extremely important modes of acceleration and run-out from the point of view of diagnostics of wheeled vehicles. However, in order to determine the traction qualities, the dependence of the traction force on their driving wheels on the speed is necessary. Therefore, for a qualitative analysis of the traction properties of wheeled vehicles, it is necessary to monitor their functioning both in steady-state modes and in the "acceleration-run-out" mode. This implies both individual application of these modes and their reasonable combination.

Analysis of existing methods and kinematic schemes of the stands that implement them shows that today there is no equipment that can measure the traction forces on each driving wheel of wheeled vehicles, and at the same time ensure the use of power and dynamic methods both individually and jointly.

Thus, the development of hybrid (inertial-force) stands that implement these methods and test modes becomes relevant. They allow you to load the car as it functions in real operating conditions. It is very important to measure, first of all, the power parameters. Power parameters are much more informative and stable than time and kinematic parameters-path and speed.

\section{Results}

In order to solve this problem, a prototype hybrid stand was developed, which is a good alternative to existing inertial and power stands, since it implements power and dynamic methods and test modes (fig. 5). The hybrid stand allows you to measure the forces and power on the driving wheels and with them, the time and kinematic parameters. The use of a hybrid stand allows you to get very reliable and stable diagnostic results, providing a more informative and correct control of the technical condition of the power plant, transmission and systems of cars with petrol or diesel engines, as well as electric vehicles and unmanned wheeled vehicles. The stand consists of a frame, two blocks of support rollers 4, kinematically connected to each other, as well as flywheels 1 and contactless sensors 10 for measuring forces on the wheels of the vehicle. One of the support roller blocks is connected to the eddy current brake 11 . The electrodynamic brake 11 of the hybrid stand is installed balancer-wise, with its own sensor 12, according to which the force loading the power plant and the transmission of the vehicle is measured. The support rollers of the stand 4 and the rollers of the tracking systems 7 have sensors 3 and 8 , which measure the speed of rotation 
of the car wheels and the speed of rotation of the support rollers. Sensor signals are processed and the stand is controlled by the power unit 14 and the amplifier and converter unit 13 .

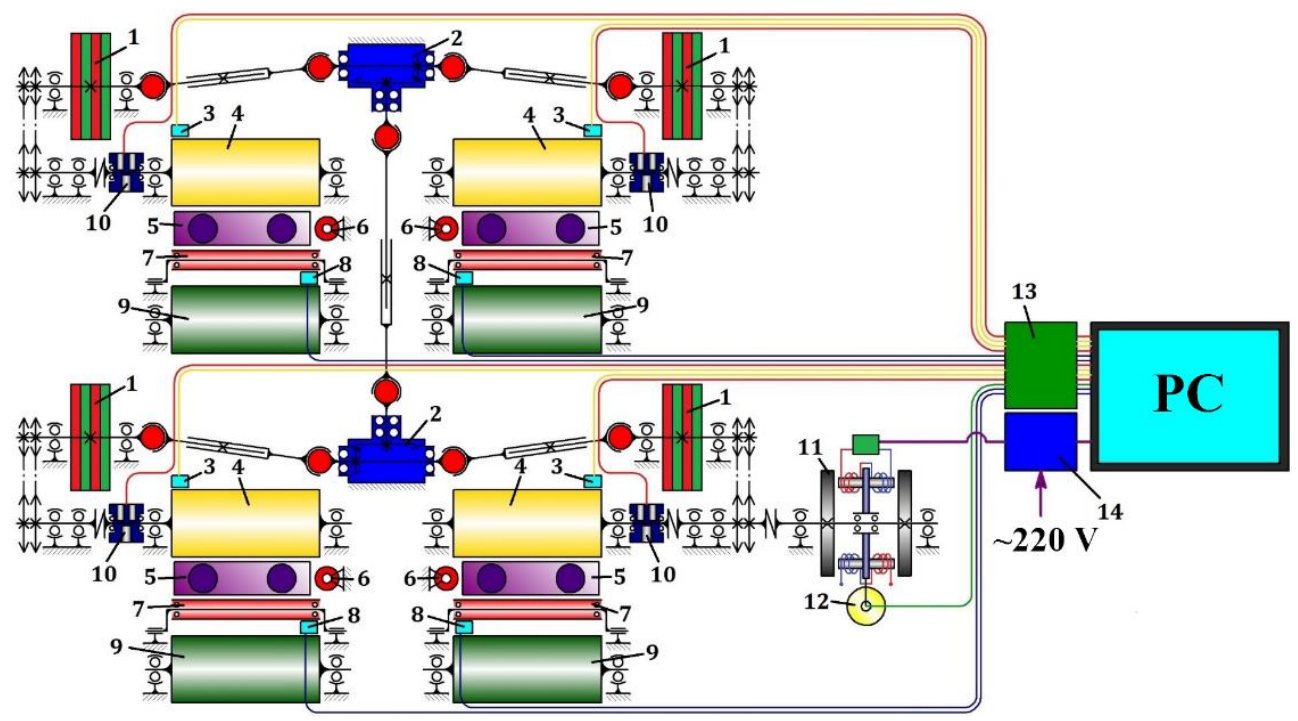

Fig. 5. Hybrid stand:

1-flywheel; 2-reducer; 3-speed sensor of stand rollers; 4-support roller; 5-lifting device;

6-bump roller; 7-tracking roller; 8-wheel speed sensor; 9-supporting roller;

10-non-contact force sensor; 11-electrodynamic brake; 12 -force sensor on the brake;

13-amplifier and converter unit; 14-power unit

As already mentioned, non-contact magnetoelastic sensors, 10 are installed on the drive shafts of each pair of support rollers 4 to measure the forces on each wheel [11]. The stand allows you to monitor both the traction and braking properties of cars in both acceleration and steady-state mode, measuring the forces on each wheel individually. It is able to diagnose anti-lock, anti-slip and other intelligent vehicle systems, as well as their traction properties, power plant and transmission.

On the hybrid stand, trial tests of the KIA SOUL EV electric car were carried out in the acceleration mode, under the action of a constant loading force from the stand's brake. The graph of this process shows the simultaneous effect on the electric vehicle of both dynamic and power test modes of loading the power plant and transmission of the tested electric vehicle (fig. 6). 


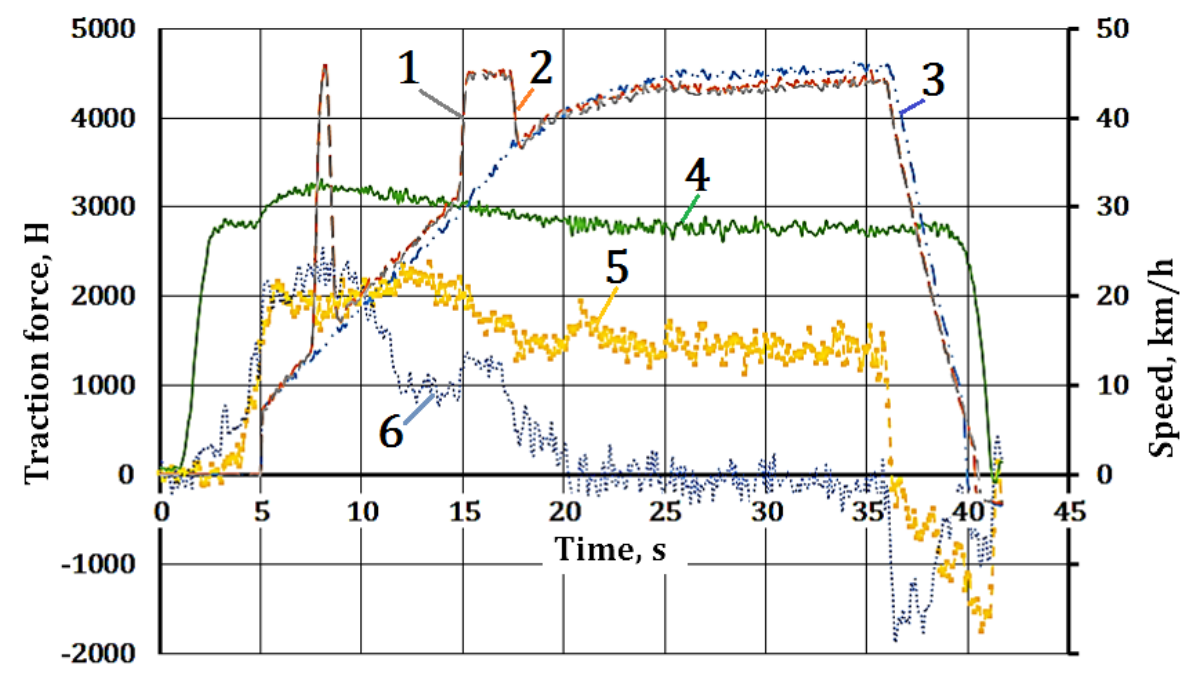

Fig. 6. Acceleration of the KIA SOUL EV electric car at the hybrid stand:

1 - left wheel speed; 2-right wheel speed; 3-stand roller speed; 4-resistance force;

5 -force on the right wheel; 6-force on the left wheel.

The graph shows the speed of rotation of the wheels of an electric vehicle on the stand rollers, the traction forces on each of its driving wheels, and the loading force with which the electrodynamic brake seeks to stop its wheels. The forces measured on the wheels of the car are unstable, due to the occurrence of torsional vibrations in the transmission of the electric vehicle, its wheels and the Flyweight of the stand. This problem has yet to be resolved.

\section{Conclusion}

It is proved that in order to provide highly informative and stable test modes of operation of a wheeled vehicle, it is necessary to use hybrid (inertia-force) stands during their diagnostics. They allow you to perform technical condition monitoring and diagnostics of vehicle power plants using both power and dynamic methods and measure both power and kinematic parameters on each driving wheel.

Hybrid stands and the methods they implement allow:

1. monitor the technical condition of power plants and transmission of wheeled vehicles, taking into account power losses in the transmission, as well as speed and power losses in the tires of the driving wheels;

2. optimize the standard values of test modes for each type of wheeled vehicles;

3. to optimize the scan parameters, such as power and kinematic, and establish their normative values;

4. develop a methodology for monitoring traction qualities and diagnostics of electric and unmanned wheeled vehicles on stands with running drums;

5. perform monitoring of anti-lock, anti-slip and intelligent vehicle systems.

\section{Reference}

1. L. Yao, Y. Xiao, X. Gong, J. Hou, X. Chen, Journal of Power Sources, 453, 10 (2020) https://doi.org/10.1016/j.jpowsour.2020.227870 
2. V. Larsson, R. Arvidsson, A. Westerlund, N, Åkerblom, IFAC-PapersOnLine, 49, p. 141-146, (2016) https://doi.org/10.1016/j.ifacol.2016.08.022

3. Y. Kang, B. Duan, Z. Zhou, Y. Shang, C. Zhang, Applied Energy, 259, 13 (2020) https://doi.org/10.1016/j.apenergy.2019.114170

4. Y. Wanga, J. Tiana, Z. Chena, X. Liub, Measurement, 131, p. 443-451 (2019) https://doi.org/10.1016/j.measurement.2018.09.007

5. I. Kleilat, H. Al-Sheikh, N. Moubayed, G.Hoblos, IFAC-PapersOnLine, 51, p.326-331 (2018) https://doi.org/10.1016/j.ifacol.2018.09.597

6. Z. Wang, J. Hong, P. Liu, L. Zhang, Applied Energy, 196, p.289-302 (2017) https://doi.org/10.1016/j.apenergy.2016.12.143

7. S. Vorobyov, I. Chernyaev, V. Nazarkin, K. Filippov, Transportation Research Procedia, 20, p.695-701 (2017) https://doi.org/10.1016/j.trpro.2017.01.113

8. J. Ejsmont, W .Owczarzak, Measurement, 145, p.144-149 (2019) https://doi.org/10.1016/j.measurement.2019.05.071

9. M. Pexaa, D. Madera, J. Čedíka, B. Peterkaa, M. Müllerb, P. Valášekb, S. Hlochc, Measurement, 152, 20 (2020) https://doi.org/10.1016/j.measurement.2019.107287

10. A. Fedotov, S. Krivtsov, O. Yankov, Proc. of the Intern. Conf. AVENT, 1, p.147-151 (2018) DOI:10.2991/avent-18.2018.28

11. O. Yan'kov, A. Chernyshkov, M. Kornyakov, A. Gilev, IOP Conf. Series: Materials Science and Engineering, 632, 15 (2019) DOI:10.1088/1757-899X/632/1/012025 